\title{
MIOMECTOMÍA ABDOMINAL VERSUS MIOMECTOMÍA LAPAROSCÓPICA: EXPERIENCIA PRELIMINAR DEL HOSPITAL CLIINICO DE LA PONTIFICIA UNIVERSIDAD CATÓLICA DE CHILE
}

\author{
Milena Zamboni T. ${ }^{1}$, María Isabel Sigüenza V. ${ }^{2}$, Cristián Pomés C. ${ }^{1}$, Mauricio \\ Cuello F. ${ }^{1}$ \\ ${ }^{1}$ Departamento de Obstetricia y Ginecología, ${ }^{2}$ Programa en Obstetricia y Ginecología, Facultad de Medicina, Pontificia \\ Universidad Católica de Chile.
}

\section{RESUMEN}

Antecedentes: Múltiples pacientes requieren un manejo conservador del mioma uterino. Muchas de ellas no han completado su paridad, y otras no desean la remoción del útero preocupadas del impacto que esa alternativa tenga en su vida sexual. Objetivos: Evaluar nuestra experiencia en pacientes miomectomizadas mediante laparoscopia o laparotomía y revisar la literatura para definir las ventajas de cada técnica y recomendaciones para su indicación. Método: Estudio prospectivo, no randomizado, de pacientes miomectomizadas en el Servicio de Ginecología del Hospital Clínico de la Universidad Católica de Chile, durante el período noviembre de 2007 y mayo de 2008. Resultados: La población incluida fue de 43 casos; 16 casos correspondieron a miomectomía laparoscópica $(37,2 \%)$ y 27 a miomectomía por laparotomía $(67,8 \%)$. La miomectomía por laparotomía se realizó en $40 \%$ de los casos utilizando una incisión transversa mínima. Esta vía de abordaje fue más frecuentemente utilizada cuando se trataba de miomas de gran tamaño o de múltiples miomas. En nuestra serie, la miomectomía laparoscópica se asociaría a un menor requerimiento de analgesia. No hay diferencias en la frecuencia de complicaciones, tiempo operatorio y estadía hospitalaria entre ambas técnicas, cuando se trata de miomas operados menores de $5 \mathrm{~cm}$. Conclusión: Nuestros resultados sugieren que la miomectomía por laparotomía es una técnica segura que debiese privilegiarse cuando se trata de miomas grandes y múltiples. La laparoscopia es igualmente segura reservada para casos bien seleccionados y cirujanos experimentados en el procedimiento.

\section{PALABRAS CLAVE: Mioma, miomectomía, cirugía, laparoscopia, complicaciones}

\section{SUMMARY}

Background: Multiple patients ask for a conservative management of uterine fibroids. Some of the patients have not completed their parity at the time of surgery, and others do not want an hysterectomy worried about de impact of this procedure in their sexual life. Objectives: The objective of this study was to evaluate our experience in the short term surgical results of patients undergoing a laparoscopic or abdominal myomectomy. We also did a literature review to define the advantages for each technique and their indications. Methods: A prospective, non-randomized study comparing short term surgical outcomes in patients undergoing laparoscopic versus abdominal myomectomy in the Gynecology Service of the Clinical Hospital of the Pontificia Universidad Católica de Chile, between November 2007 and May 2008. Results: Forty-three patients undergoing myomectomy in our centre were analyzed. 16 of them were approached by laparoscopy (37.2\%) 
and 27 by laparotomy ( $67.8 \%)$. In $40 \%$ of them, abdominal myomectomy was performed using a minimal transverse incision. This approach was more frequently used when treating larger size $(>5 \mathrm{~cm})$ or multiple myomas. In our study, laparoscopic myomectomy was associated with less requirements of analgesia. No differences in complication rates, operative time, and hospital stay were observed between techniques in myomas smaller than $5 \mathrm{~cm}$. Conclusions: Our results indicate that abdominal myomectomy is a safe technique that should be used in cases of larger size or multiple myomas. Laparoscopy is equally safe but should be reserved for well-chosen cases and performed by experienced laparoscopic surgeons.

\section{KEY WORDS: Fibroids, myomectomy, surgery, laparoscopy complications}

\section{INTRODUCCIÓN}

El mioma uterino es la neoplasia benigna más común del aparato genital femenino. Su prevalencia es variable dependiendo del origen racial. En los países con mayor porcentaje de población afro-americana la prevalencia alcanza el $50 \%$ del total de mujeres (1). La sintomatología que origina consulta asociada a esta entidad, habitualmente se manifiesta durante los últimos años de vida reproductiva de la mujer, siendo la hipermenorrea y la anemia secundaria junto con el algia pelviana por compresión de estructuras vecinas secundario al tamaño que adquiere el tumor (1). Con el advenimiento de la ultrasonografía pelviana y su uso frecuente como parte de la evaluación rutinaria, se ha producido un incremento significativo de casos, diagnosticados en etapa asintomática, generando nuevas necesidades de manejo y seguimiento de la enfermedad (2).

El manejo tradicional de esta patología involucró la cirugía asociada a la remoción del cuerpo uterino o el útero completo. Históricamente, esa decisión, no constituyó un problema, dado que la mayoría de los casos se presentaban en pacientes sin deseos de paridad futura. Sin embargo, en las últimas dos décadas, un cambio sustantivo ha ocurrido en el perfil de las mujeres afectadas por esta enfermedad. Muchas de ellas no han tenido hijos al momento del diagnóstico o no han completado su paridad, y otras no desean la remoción del útero preocupadas del impacto que dicha alternativa tenga en su vida sexual (3).

Afortunadamente, los avances tecnológicos, tanto en el diagnóstico como en el manejo, han permitido desarrollar nuevas modalidades de tratamiento que permiten la conservación del útero en mujeres afectadas por miomas. En pacientes con deseos de fertilidad futura, las opciones más usadas son la miomectomía con o sin uso previo de agonistas GNRH y para aquellas que desean preservar su útero, además de la miomectomía, la embolización de las arterias uterinas, el ultrasonido focalizado, la ablación endometrial y la miolisis (2). Específicamente, en nuestro país, existen pocos trabajos que hayan analizado los resultados obtenidos con el manejo quirúrgico conservador del mioma uterino en cuanto a complicaciones derivadas de la cirugía y fertilidad futura. Tampoco se ha evaluado los resultados de la miomectomía laparoscópica comparado con la miomectomía realizada por laparotomía.

El objetivo de este estudio es evaluar nuestra experiencia en los resultados quirúrgicos inmediatos de pacientes miomectomizadas mediante laparoscopia o laparotomía y revisar la literatura con el fin de definir las ventajas de cada técnica y recomendaciones para su indicación.

\section{PACIENTES Y MÉTODO}

Estudio prospectivo no randomizado de pacientes miomectomizadas en el Servicio de Ginecología del Hospital Clínico de la Universidad Católica de Chile, durante el período comprendido entre noviembre de 2007 y mayo de 2008.

Se incluyeron todas las pacientes con diagnóstico preoperatorio de mioma uterino sintomático (hipermenorrea, algia pelviana o síntomas compresivos) o que el mioma fuese causa de infertilidad. Se excluyó los casos de mioma asintomático (hallazgo de la ultrasonografía), los casos en que se practicó una miomectomía como parte de una cirugía motivada por otra causa y aquellos casos con tratamiento médico previo. En todos los casos hubo confirmación histológica del diagnóstico de leiomioma uterino por un patólogo del centro.

Las variables estudiadas fueron: edad de la paciente, tamaño individual del mioma, peso total, número de miomas extirpados, vía de abordaje (laparotomía versus laparoscopia), necesidad de conversión, tiempo operatorio, complicaciones durante la cirugía y en el período postoperatorio inmediato (primeras 72 horas), modalidad y tiempo de 
analgesia postoperatoria y la duración de la estadía hospitalaria.

Para comparar los resultados quirúrgicos a corto plazo entre miomectomía abierta (MA) y laparoscópica (ML), se definió que las dos técnicas quirúrgicas tienen asociadas la misma probabilidad de éxito. Se excluyó del análisis los casos de miomectomía con abordaje combinado (vía abdominal o laparoscópica con tiempo de resectoscopia) o aquellos casos de mioma submucoso primariamente tratados con resectoscopia. Para el análisis estadístico se realizó las pruebas de Chi cuadrado $\left(x^{2}\right)$, t-test o Wilcoxon, fijando como diferencia significativa un valor $p<0,05$.

\section{RESULTADOS}

Se reclutaron 52 casos de miomectomía. De ellas, se excluyeron 5 casos, 2 por tratarse de miomectomías con abordaje combinado, 2 por miomectomías mediante resectoscopia y 1 caso por torsión de mioma pediculado. Finalmente, otros 4 casos se excluyeron por concomitancia a otra enfermedad (endometriosis). Así la población incluida en el análisis se redujo a 43 casos. De ellos, 16 casos correspondieron a miomectomía laparoscópica $(37,2 \%)$ y 27 casos a miomectomía por laparotomía $(67,8 \%)$.

La edad promedio de las pacientes fue $33,7 \pm$ 7,5 años, con mediana de 37 años. El percentil 25 y 75 fueron 33 y 40 años respectivamente. La mayoría de las pacientes eran nulíparas (70\%); en la Tabla I se desglosa la distribución por paridad.

El rango de miomas extirpados según abordaje fue: 1 a 5 miomas por vía laparoscópica versus 1 a 21 miomas por vía abdominal. El promedio de miomas extirpados por laparotomía fue significativamente mayor que el observado por laparoscopia $(3,6 \pm 4,9$ versus $1,7 \pm 1,1$ miomas, $t$ test $p<0,03)$. La Tabla II resume la distribución del número de miomas extirpados por paciente.

\section{Tabla I}

\section{DISTRIBUCIÓN SEGÚN PARIDAD DE LA MUESTRA ESTUDIADA}

\begin{tabular}{lr}
\hline Paridad previa & $\mathrm{n}(\%)$ \\
\hline Nulípara & $28(70)$ \\
Multípara 1 & $5(12,5)$ \\
Multípara 2 & $6(15)$ \\
Multípara 3 & $1(2,5)$ \\
\hline Total & $40(100)$
\end{tabular}

El tamaño promedio del mioma más grande extirpado fue también significativamente mayor en el grupo operado por laparotomía $(7,1 \pm 3,5$ $\mathrm{cm}$ versus $4,3 \pm 2,1 \mathrm{~cm}$, t-test $\mathrm{p}<0,002$ ). Aunque

\section{Tabla II}

\section{DISTRIBUCIÓN DEL NÚMERO DE MIOMAS EXTIRPADOS POR PACIENTE}

\begin{tabular}{cc}
\hline Miomas extirpados & $\mathrm{n}(\%)$ \\
\hline 1 & $24(57,1)$ \\
$2-3$ & $10(23,8)$ \\
$4-5$ & $5(11,9)$ \\
6 & $3(7,1)$ \\
\hline Total & $42(100)$
\end{tabular}

no en todos los casos se registró el peso del total de miomas extirpados (particularmente en el grupo operado por laparoscopia), el peso promedio fue significativamente mayor en el grupo operado por laparotomía $(243,9 \pm 322,8 \mathrm{~g}$ versus $46 \pm 16,1 \mathrm{~g}$, t-test $p<0,006)$.

El promedio de tiempo operatorio en las pacientes sometidas a miomectomía vía laparotomía fue $80,6 \pm 29,1$ minutos (rango: 35 a $180 \mathrm{~min}$ ) versus $108,1 \pm 53,1$ minutos (rango: 50 a $240 \mathrm{~min}$ ) en las pacientes operadas por vía laparoscópica, diferencia no significativa. Sin embargo, al analizar sólo los casos de mioma uterino con tamaño menor de $5 \mathrm{~cm}$, observamos que la diferencia se hace significativa en favor de la miomectomía por laparotomía $(61,7 \pm 16 \mathrm{~min}$ versus $113 \pm 3 \mathrm{~min}$, Wilcoxon, $\mathrm{p}<0,04)$.

No se registraron complicaciones perioperatorias mayores en ninguno de los dos grupos estudiados. En el caso de la miomectomía laparoscópica hubo un caso en que fue necesaria la conversión a laparotomía; se trataba de una paciente con un mioma de $5 \mathrm{~cm}$ ubicado en la cara posterior del útero y en donde el cirujano consideró como técnicamente difícil el abordaje por laparoscopia. Hubo sólo un caso en que fue necesario realizar una transfusión intraoperatoria producto de sangrado mayor. Correspondió a una paciente del grupo operado por laparotomía; se trataba de una paciente con miomas múltiples entre 1,3 y $12 \mathrm{~cm}$ de diámetro que estaba siendo sometida a una miomectomía múltiple. No hubo complicaciones mayores en el postoperatorio o necesidad de reexploración quirúrgica.

Todas las pacientes, independiente de la vía de abordaje recibieron un esquema basado en an- 
tiinflamatorios no esteroidales administrados por vía endovenosa o por bomba de infusión continua. No hubo diferencias entre los grupos respecto a la selección de este esquema basal. Al no observar diferencias en el esquema basal se decidió evaluar los requerimientos adicionales de analgesia. El esquema de rescate consistió en bolos de morfina endovenosa previo a indicar analgesia controlada por el paciente (PCA). Hubo un porcentaje significativamente mayor de casos en que se indicó bolos de morfina en el grupo de pacientes operadas por laparotomía (36\% versus $\left.28 \%, x^{2} p<0,05\right)$.

La estadía fue significativamente menor en el grupo operado por laparoscopia $(1,93 \pm 0,68$ días versus $3,1 \pm 0,9$ días, t-test $p<0,0001)$. No hubo diferencia significativa si se compara las miomectomías con tamaño del mioma menor de $5 \mathrm{~cm}$. La Tabla III resume el número de días de hospitalización.

En las pacientes operadas por laparotomía, la incisión abdominal fue transversal suprapúbica, ocupándose técnica mínimamente invasiva (incisión de Pelosi o minilaparotomía) en 11 de ellas

\section{Tabla III \\ DISTRIBUCIÓN DEL NÚMERO DE DÍAS DE HOSPITALIZACIÓN SEGÚN VÍA DE ABORDAJE DE LA MIOMECTOMÍA}

\begin{tabular}{lll}
\hline $\begin{array}{c}\text { Hospitalización } \\
\text { (días) }\end{array}$ & $\begin{array}{c}\text { Laparotomía } \\
\mathrm{n}(\%)\end{array}$ & $\begin{array}{c}\text { Laparoscopia } \\
\mathrm{n}(\%)\end{array}$ \\
\hline 1 & $0(0)$ & $4(26,7)$ \\
2 & $6(22,2)$ & $9(60)$ \\
3 & $16(59,3)$ & $2(13,3)$ \\
4 & $5(18,5)$ & $0(0)$ \\
\hline Total & $27(100)$ & $15(100)$
\end{tabular}

(40,7\%). En $3(6,3 \%)$ pacientes miomectomizadas por laparoscopia fue necesario ampliar la incisión suprapúbica para extraer la pieza.

\section{DISCUSIÓN}

El análisis de los resultados demuestra que es posible realizar de manera segura una miomectomía única como múltiple ya sea por vía laparotomía o por laparoscopia. En esta serie no se registraron complicaciones mayores. Históricamente datos no publicados de nuestro grupo demuestran que la necesidad de histerectomía o de reexploración ulterior por sangrado uterino mayor es excepcional. Sólo 3 casos se registran en los últimos 10 años, y correspondieron a casos de miomectomía múltiple, abordada por laparotomía, en que el tamaño de más de un mioma superaba los $5 \mathrm{~cm}$ de diámetro mayor. Estos resultados son concordantes con series internacionales (4-10).

A fin de garantizar el buen resultado del manejo conservador mediante miomectomía, tal como lo demuestra esta serie, es importante destacar algunas recomendaciones a seguir. En primer lugar es esencial realizar una evaluación preoperatoria completa. Además de una adecuada anamnesis, donde se debe buscar en forma dirigida los síntomas que avalan la indicación quirúrgica, es imprescindible realizar un hemograma para la pesquisa de anemia secundaria. Nunca debe omitirse el examen bimanual ya que permite establecer el tamaño del útero, elemento a considerar al momento de elegir la vía de abordaje. Siempre es recomendable realizar algún estudio de imágenes complementario; esto permite definir el número de miomas, su tamaño y localización (submucoso, intramural, subseroso) constituyendo un elemento de juicio al momento de decidir la vía de abordaje (1). En pacientes en que existe historia de metrorragia, siempre se debe considerar una biopsia endometrial para descartar patología tumoral del endometrio, la cual se realiza de forma preferente en el preoperatorio, ya que ayudara a decidir tanto el tipo de cirugía (miomectomia vs histerectomía) y el equipo adecuado para efectuar la operación (equipo de ginecología general o equipo de oncología ginecológica).

En esta serie, desde un punto de vista técnico, la miomectomía por laparoscopia no ofrece ventajas sobre la miomectomía por laparotomía. Al analizar distintas variables, tales como tamaño de miomas, número y localización de miomas extirpados, tiempo operatorio e incidencia de complicaciones, no encontramos diferencias en favor de la laparoscopia.

De acuerdo a nuestros resultados, en los casos de miomectomía por laparotomía el tiempo quirúrgico es significativamente menor, se extirpan más miomas habitualmente y son más grandes. Junto a ello no hay mayor número de complicaciones. Así también, aunque observamos un menor requerimiento de analgesia con opiáceos y una menor estadía hospitalaria en pacientes operadas por laparoscopia, las diferencias se minimizan o desaparecen cuando se comparan miomas que miden menos de $5 \mathrm{~cm}$. Estos resultados contrastan con los resultados de algunas series internacionales en donde se demuestra los beneficios de la laparoscopia en términos de dolor postoperatorio, pérdidas hemáticas, estadía hospitalaria y tiempo de recupe- 
ración (4,7,8,11-15).

La falta de beneficio de la laparoscopia en nuestra serie puede explicarse por dos razones. La primera se refiere a la poca experiencia de algunos de nuestros cirujanos con esta vía de abordaje (cabe recordar que se trata de un centro con programa de formación de postgrado) y a la mayor familiaridad con técnicas de miomectomía por laparotomía. A ello se agrega la introducción contemporánea de técnicas de laparotomía mínimamente invasivas las cuales han homologado la evolución postoperatoria entre ambos grupos.

En nuestra serie el $40 \%$ de los casos de laparotomía usó dichas técnicas (minilaparotomía) haciendo que el tiempo quirúrgico, los requerimientos de analgesia y la estadía hospitalaria fuesen similares o mejores para este grupo versus las pacientes tratadas por laparoscopia. Ello se hace más evidente cuando se compara pacientes operadas por mioma menor de $5 \mathrm{~cm}$. Cabe señalar, que nuestros resultados por laparoscopia son comparables a series internacionales en cuanto a tiempo quirúrgico y tasa de complicaciones (14,16-18). Recientemente se publicó un estudio randomizado multicéntrico comparando la miomectomía laparoscópica con la realizada por minilaparotomía, que demostró beneficios a favor de la minilaparotomía en términos del tiempo de enucleación del mioma y número de histerotomías. Sin embargo, la laparoscopia fue mejor en términos de pérdidas hemáticas durante la cirugía, de las variaciones en los niveles de hemoglobina, del uso de drogas analgésicas en el postoperatorio y del tiempo de hospitalización (19). Pareciera, de acuerdo a la evidencia disponible, que ambas técnicas ofrecen ventajas y desventajas, haciendo que la elección dependa principalmente de la experiencia del cirujano y de la complejidad del caso particular.

De acuerdo a la experiencia de nuestro grupo, es posible actualmente realizar miomectomía laparoscópica en manos de expertos, en aquellas casos en que el número de miomas no supera 5 y el tamaño del mayor no supera los 7 a $8 \mathrm{~cm}$. Sin embargo, una consideración adicional importante al momento de elegir esta vía es la ubicación anatómica de cada uno de ellos. Tal consideración busca evitar incisiones uterinas múltiples, minimizar el sangrado y evitar la conversión a laparotomía.

Al revisar series internacionales encontramos que los criterios de selección son variables y no exentos de controversia. Algunos autores definen la vía de abordaje de acuerdo el número de miomas presentes. En esos trabajos se opta por la laparotomía si el número supera los 2 a 4 miomas $(8,20,21)$ o se basan en el diámetro del mioma más grande, inclinándose por la laparotomía si el mioma excede los $6(8,22)$ a $10 \mathrm{~cm}(21)$. Dubuisson y cols (23), definieron establecer un modelo predictivo para el riesgo de conversión, incluyendo 4 factores: mioma de tamaño mayor a $5 \mathrm{~cm}$ a la ultrasonografía (OR 10,3; IC 95\%: 2,8-37,9); localización intramural (OR: 4,3; IC 95\%: 1,3-14,5), un mioma localización anterior (OR 3,4; IC 95\%: 1,3-9,0) y el uso preoperatorio de agonistas de GNRH (OR: 5,4; IC 95\%: 2,0-14,2). En nuestra serie, la única conversión se registró en el caso de una mujer con un mioma de 5 $\mathrm{cm}$ en cara posterior.

Además de ser realizada por gente entrenada y con experiencia en laparoscopia, algunas consideraciones técnicas deben tenerse presente al momento de inclinarse por una miomectomía por vía laparoscópica. Dichas consideraciones permiten minimizar las complicaciones asociadas (pérdidas hemáticas excesivas, necesidad de conversión, etc.) y maximizar el éxito reproductivo futuro. Dentro de ellas están: ubicar los sitios de entrada considerando la necesidad de sutura endoscópica (24), la infiltración local con vasopresina previo a la extirpación del mioma (25-27), limitar el número de incisiones (longitudinales u oblicuas, pero siempre considerando los reparos anatómicos y el facilitar la sutura posterior del lecho) $(5,20,28,29)$, suturar el miometrio en múltiples planos (especialmente en pacientes con deseo de fertilidad futura) y elegir la vía de extracción dependiendo del caso particular (mioma pequeño: a través del trócar, miomas de mayor tamaño: uso de morcelador o extracción por colpotomía posterior) .

Cuando se opta por la vía abierta, a fin de obtener resultados similares es útil tener en cuenta el uso de minilaparotomía y de separadores plásticos expandibles para minimizar el dolor asociado al trauma de la herida, la infiltración previa con vasoconstrictores o la aplicación de ligaduras transitorias a nivel del istmo y el uso de las mismas técnicas de incisión y sutura descritas para la laparoscopia.

El seguimiento de esta serie no permite evaluar los resultados a largo plazo de cada técnica. Al menos a la fecha, podemos comentar que no hemos tenido diferencias significativas en términos de recurrencia. Ello es comparable a lo reportado en series internacionales donde las tasas de recurrencia son similares entre ambas técnicas (alrededor del 20 a $30 \%$ de los casos) (30).

\section{CONCLUSIÓN}

Nuestros resultados permiten concluir que es posible realizar en forma segura miomectomía laparoscópica en casos bien seleccionados y efectuada 
por cirujanos expertos. La miomectomía por laparotomía es una alternativa del todo comparable que otorga beneficios similares cuando se introducen técnicas mínimamente invasivas, y que debe ser privilegiada para la mayoría de los casos, particularmente cuando se trata de cirujanos con poca experiencia en laparoscopia.

\section{BIBLIOGRAFÍA}

1. Parker WH. Etiology, symptomatology, and diagnosis of uterine myomas. Fertil Steril 2007;87:725-36.

2. Parker WH. Uterine myomas: management. Fertil Steril 2007;88:255-71.

3. Berger U, Altgassen C, Kuss S, Schneider A. Patients' satisfaction with laparoscopic myomectomy. J Psychosom Obstet Gynaecol 2006;27:225-30.

4. Cagnacci A, Pirillo D, Malmusi S, Arangino S, Alessandrini C, Volpe A. Early outcome of myomectomy by laparotomy, minilaparotomy and laparoscopically assisted minilaparotomy. A randomized prospective study. Hum Reprod 2003;18:2590-4.

5. Dubuisson JB, Chapron C, Levy L. Difficulties and complications of laparoscopic myomectomy. J Gynecol Surg 1996;12:159-65.

6. Hasson HM, Rotman C, Rana N, Sistos F, Dmowski WP. Laparoscopic myomectomy. Obstet Gynecol 1992;80:884-8.

7. Holzer A, Jirecek ST, Illievich UM, Huber J, Wenzl RJ. Laparoscopic versus open myomectomy: a doubleblind study to evaluate postoperative pain. Anesth Analg 2006;102:1480-4.

8. Mais V, Ajossa S, Guerriero S, Mascia M, Solla E, Melis GB. Laparoscopic versus abdominal myomectomy: a prospective, randomized trial to evaluate benefits in early outcome. Am J Obstet Gynecol 1996;174:6548.

9. Nezhat C, Nezhat F, Silfen SL, Schaffer N, Evans D. Laparoscopic myomectomy. Int J Fertil 1991;36:27580.

10. Verkauf BS. Myomectomy for fertility enhancement and preservation. Fertil Steril 1992;58:1-15.

11. Alessandri F, Lijoi D, Mistrangelo E, Ferrero S, Ragni N. Randomized study of laparoscopic versus minilaparotomic myomectomy for uterine myomas. J Minim Invasive Gynecol 2006;13:92-7.

12. Sesti F, Capobianco F, Capozzolo T, Pietropolli A, Piccione E. Isobaric gasless laparoscopy versus minilaparotomy in uterine myomectomy: a randomized trial. Surg Endosc 2008;22:917-23.

13. Tan J, Sun Y, Dai H, Zhong B, Wang D. A randomized trial of laparoscopic versus laparoscopic-assisted minilaparotomy myomectomy for removal of large uterine myoma: short-term outcomes. J Minim Invasive Gynecol 2008;15:402-9.

14. Malzoni M, Rotond M, Perone C, Labriola D, Ammaturo F, Izzo A, Panariello S, Reich H. Fertility after laparoscopic myomectomy of large uterine myomas: operative technique and preliminary results. Eur J Gynaecol Oncol 2003;24:79-82.

15. Hurst BS, Matthews ML, Marshburn PB. Laparoscopic myomectomy for symptomatic uterine myomas. Fertil Steril 2005;83:1-23.

16. Andrei B, Crovini G, Rosi A. Uterine myomas: pelviscopic treatment. Clin Exp Obstet Gynecol 1999;26:446.

17. Landi S, Zaccoletti R, Ferrari L, Minelli L. Laparoscopic myomectomy: technique, complications, and ultrasound scan evaluations. J Am Assoc Gynecol Laparosc $2001 ; 8: 231-40$.

18. Altgassen C, Kuss S, Berger U, Loning M, Diedrich K, Schneider A. Complications in laparoscopic myomectomy. Surg Endosc 2006;20:614-8.

19. Palomba S, Zupi E, Russo T, Falbo A, Marconi D, Tolino A, Manguso F, Mattei A, Zullo F. A multicenter randomized, controlled study comparing laparoscopic versus minilaparotomic myomectomy: short-term outcomes. Fertil Steril 2007;88:942-51.

20. Milad M, Sankpal S. Laparoscopic approaches to uterine leiomyomas. Clin Obstet Gynecol 2001;44:40111.

21. Miller CE, Johnston M, Rundell M. Laparoscopic myomectomy in the infertile woman. J Am Assoc Gynecol Laparosc 1996;3:525-32.

22. Parker WH, Rodi IA. Patient selection for laparoscopic myomectomy. J Am Assoc Gynecol Laparosc 1994;2:23-6.

23. Dubuisson JB, Fauconnier A, Fourchotte V, BabakiFard K, Coste J, Chapron C. Laparoscopic myomectomy: predicting the risk of conversion to an open procedure. Hum Reprod 2001;16:1726-31.

24. Koh C, Janik G. Laparoscopic myomectomy: the current status. Curr Opin Obstet Gynecol 2003;15:295301.

25. Agostini A, Ronda I, Franchi F, Bretelle F, Roger V, Cravello L, Blanc B. Oxytocin during myomectomy: a randomized study. Eur J Obstet Gynecol Reprod Biol 2005;118:235-8.

26. Kongnyuy EJ, Wiysonge CS. Interventions to reduce haemorrhage during myomectomy for fibroids. Cochrane Database Syst Rev 2007: CD005355.

27. Wang CJ, Lee CL, Yuen LT, Kay N, Han CM, Soong YK. Oxytocin infusion in laparoscopic myomectomy may decrease operative blood loss. J Minim Invasive Gynecol 2007; 14:184-8.

28. Dubuisson JB, Chapron C, Fauconnier A, Kreiker G. Laparoscopic myomectomy and myolysis. Curr Opin Obstet Gynecol 1997;9:233-8.

29. Igarashi M. Value of myomectomy in the treatment of infertility. Fertil Steril 1993;59: 1331-2; author reply 1332-3.

30. Rossetti A, Sizzi O, Soranna L, Cucinelli F, Mancuso S, Lanzone A. Long-term results of laparoscopic myomectomy: recurrence rate in comparison with abdominal myomectomy. Hum Reprod 2001;16:770-4. 